\title{
Über die Löslichkeit des Arseniks und den Molekularzustand seiner Lösung.
}

Von

L. Bruner und St. Toefoczko.

Bei einer umfassenden Untersuchung über die Auflösungsgeschwindigkeit fester Körper haben wir einen Apparat ${ }^{1}$ in Anwendung gebracht, der uns gestattete, eine äufserst kräftige Rührung (ca. 2000 Umdrehungen pro Minute) des Lösungsgemisches zustande zu bringen. Da wir neben den hauptsächlichen Messungen über die Auflösungsgeschwindigkeit des Alabasters und Marienglases $\left(\mathrm{CaSO}_{4} .2 \mathrm{H}_{2} \mathrm{O}\right)$ auch einige Messungsreihen über die arsenige Säure ausgefübrt haben, so haben wir auch die Löslichkeit des Arseniks für verschiedene Temperaturen gemessen. Wie in letzter Zeit genau bekannt geworden, ${ }^{2}$ ist die Auflösung des Arseniks - wohl doch wegen der eintretenden Hydratation - ein äuiserst langsamer Prozeis; die Hydratation kann zwar katalytisch sehr stark beschleunigt werden, dann ist jecloch keine volle Sicherheit, dafs auch die Löslichkeit unbeeinflufst geblieben ist. Die Langsamkeit der Auflösung ist es wohl, die die ganz enormen Unterschiede in den. älteren Angaben über die Löslichkeit des Arseniks bedingt hat. Bei dieser Sachlage ist die kräftige Durchmischung des Lösungsgemisches - wie es ron uns realisiert wurde - eine sehr günstige, wenn gerade nicht notwendige Bedingung einer exakten Löslichkeitsbestimmung.

1 Z. anory. Chem. 35, 23. Vergl. auch Bulletin de l'Acad. de Cracovie. Octobre 1903.

2 K. Drucker, Zeitschr. phys. Chem. 36, 173. L. Bruser u. St. Tomoczko Bulletin de l'Acad. de Crac., 1. c. 
Die Bestimmungen sind so ausgeführt worden, dafs in den Apparat cine grolse überschülsige Menge (ca. $70-100 \mathrm{~g}$ ) kristallisierter regulären, grobkörniger, am Schleifpapier erhaltenen, arsenigen Säure eingefüllt worden ist, mit 1 Liter Wasser übergegossen - und der schraubförmige Rührer in rasche Bewegung mit einem Luftmotor $(1 / 40 \mathrm{HP})$ versetzt. Die Tourenzahl betrug im Mittel 800-1000 pro Minute. Der ganze Apparat war in einen Thermostaten eingesenkt. dessen Temperatur in üblicher Weise reguliert wurde. Ein im Inneren des Apparates angebrachtes Thermometer zeigte unmittelbar die Temperatur des Lösungsgemisches. In passenden Zeitintervallen wurden Proben herauspipettiert, durch ein Filter, der auf der Versuchstemperatur gehalten worden ist, filtriert und der Gehalt an arseniger Säure mit Jodlösung mafsanalytisch bestimmt. Mit der Rảhrung wurde so lange fortgeschritten, bis die Proben in je zweistündigen Intervallen herausgenommen, sich von demselben Gehalte erwiesen. Die Einstellung des Gleichgewichtes erfolgt sehr langsam: jeder Versuch nahm mindestens 15-18 Stunden in Anspruch. Das Gleichgewicht wurde immer beiderseits - von einer unter- und einer übersättigten Lösung erreicht. Die Schwankungen im Titer der Lösung betrugen in sämtlichen Versuchen weniger als $1 \%$.

Die Resultate sind in der Tabelle 1 wiedergegeben. Es sind unter $C$ die Anzahl Gramm des $\mathrm{As}_{2} \mathrm{O}_{3}$ in 1 Liter gesättigter Lösung, unter $V$ die Anzahl Kubikzentimeter, die zur Herstellung einer gesättigten Lösung eines Grammäquivalents $\mathrm{As}_{2} \mathrm{O}_{3}=198 \mathrm{~g}$ notwendig sind, angegeben.

Tabelle.

Löslichkeit des kristallisierten Arseniks in $\mathrm{H}_{2} \mathrm{O}$ :

$\begin{array}{cccc}t & C & V & \frac{\Delta C}{\Delta t} \\ 2.0^{\circ} & 12.006 \mathrm{~g} & 16.492 & \\ 15.0^{\circ} & 16.566 \mathrm{~g} & 11.952 & 0.3508 \mathrm{~g} \\ 25.0^{\circ} & 20.384 \mathrm{~g} & 9.713^{1} & 0.3818 \mathrm{~g} \\ 39.8^{\circ} & 29.302 \mathrm{~g} & 6.757 & 0.6025 \mathrm{~g} \\ \text { Siedetemperatur } & C>60 \mathrm{~g} & - & \end{array}$

${ }^{1} \mathrm{H}$. Drucker findet (Zeitschr. phys. Chem. 36, 703) $V$ für $25^{\circ}$ a) 10490 , b) $9400:$ in Mittel 9945, eine von den unserigen nicht viel abweichende Zabl. In sehr verdünnter Schwefelsäure ist die Löslichkeit des Arseniks unverändert, nicht aber in der Salzsăure. 
Wie aus den Werten $\frac{\Delta c}{\Delta t}$ zu ersehen, folgt die Löslichkeit nicht linear mit der Temperatur. Es muiste somit eine emperische Gleichung höheren Grades gelten. Da wir nur 4 Punkte der Kurve bestimmt haben, haben wir die Berechnung derselben unterlassen.

Aus den mitgeteilten Löslichkeiten kann man leicht nach der integrierten VAN'T HoFrschen Gleichung der Reaktionsisochore: $q=-R T^{2} \frac{d \ln K}{d T}$ die Wärmetönung der Auflösung berechnen. Es ist nämlich:

und für diesen Fall $K=C$.

$$
q=\frac{2\left(\ln K_{1}-\ln K_{2}\right)^{1}}{1 / T_{1}-1 / T_{2}}
$$

Führt man die Berechnung aus, so erhält man:

$$
\begin{aligned}
& q_{2-15}=-3925 \mathrm{cal} . \\
& q_{15-2 \overline{5}}=-3542 \quad, \\
& q_{2 \overline{5}-40}=-4580 \quad,
\end{aligned}
$$

als molekulare Lösungswärme für die angegebenen Temperaturintervallen. Auf kalorimetrischem Wege ist unmittelbar die molekulare Lösungswärme des $\mathrm{As}_{2} \mathrm{O}_{3}$ zu 7550 cal. bestimmt worden. ${ }^{2} \mathrm{Da}$ die kalorimetrischen Messungen sich auf die mittlere Temperatur von $18^{\circ}$ beziehen, so dürfen wir wohl mit diesem kalorimetrischem Werte, das $q_{15-25}$, oder das Mittel von $q_{2-15}$ und $q_{15-25^{\circ}}=3740$ cal. vergleichen, indem wir die etwas höhere Zahl $q_{25}-40$, die für sich keinen kalorimetrischen Vergleichungswert hat, zur Zeit unberücksichtigt lassen:

Wir haben also für $\mathrm{As}_{2} \mathrm{O}_{3}$ :

$$
q=7550 \text { (beobachtet); } 3740 \text { (thermodynamisch berechnet). }
$$

Es fällt in die Augen, dafs der berechnete Wert fast absolut genau die Hälfte von dem beobachtetem beträgt. Es ist daraus zu schliefsen, dafs wir als Molekulargewicht der arsenigen Säure in wässeriger Lösung nicht $\mathrm{As}_{2} \mathrm{O}_{3}$ (oder selbstrerständlich $\mathrm{As}_{2} \mathrm{O}_{3}+$ $\mathrm{xH}_{2} \mathrm{O}$ ), sondern $1 / 2 \mathrm{As}_{2} \mathrm{O}_{3}$ (oder $1 / 2 \mathrm{As}_{2} \mathrm{O}_{3}+\mathrm{yH}_{2} \mathrm{O}$ ) anzunehmen haben. Mit anderen Worten: die säure, die durch Hydratation des

1 W. Nennst, Theoretische Chemie, II. Aufl, S. 599.

2 Tromsen, Thermochemische Untersuchungen, Bd. 2, S. 234. Siehe auch Bermaenot, Thermochemie, Vol. II. 
$\mathrm{As}_{2} \mathrm{O}_{3}$ in wässeriger Lösung entsteht, enthält nicht zwei, sondern nur ein Verbindungsgewicht Arsen. Der aus Löslichkeitsbestimmungen thermodynamisch gezogene Schlufs liefert also einen neuen Beweis für die Richtigkeit einer interessanten Arbeit von J. ZAwidzki, ${ }^{1}$ wo auf Grund ron Molekulargewichts- und Leitfähigkeitsmessungen der arsenigen Säure auch nur ein Verbindungsgewicht Arsen zugeschrieben wird. Die Übereinstimmung verschiedener zur Entscheidung berufenen Methoden verleiht diesem Schlusse hohe Beweiskraft.

' J. ZAwIDzkr, Ber. deutsoh. chem. Ges. 36, 1427.

Krakau, II. Chemisches Laboratorium der Jagell. Universität.

Bei der Redaktion eingegangen am 23. Oktober 1903. 